\title{
ON THE GLAUBERMAN CORRESPONDENCE
}

\author{
GABRIEL NAVARRO
}

(Communicated by Warren J. Wong)

\begin{abstract}
In this paper we give an elementary proof of the p-group case of Glauberman's correspondence.
\end{abstract}

\section{INTRODUCTION}

Let $p$ be a prime number.

Let $S$ be a $p$-group acting on a finite $p^{\prime}$-group $G$. Let $C=C_{G}(S)$ and $\operatorname{Irr}_{S}(G)=\left\{\chi \in \operatorname{Irr}(G) \mid \chi^{s}=\chi, \forall s \in S\right\}$.

It is well known that the map $K \rightarrow K \cap C$ defines a bijection from the set of $S$-invariant conjugacy classes of $G$ onto the set of conjugacy classes of $C[2$, 13.10].

Let $R$ be the full ring of algebraic integers in $\mathrm{C}$, let $M$ be a maximal ideal of $R$ containing $p R$, and set $F=R / M$. Let ${ }^{*}: R \rightarrow F$ the canonical homomorphism.

For $\chi \in \operatorname{Irr}(G)$, the map defined on $Z(F[G])$ by $\lambda_{\chi}(\widehat{K})=\omega_{\chi}(\widehat{K})^{*}$ is an algebra homomorphism from $Z(F[G])$ to $F$. Note that $\lambda_{\chi}(\widehat{K})=(\chi(x)|K| / \chi(1))^{*}$ for $x \in K$.

\section{TWO LEMMAS}

2.1. Lemma. Let $\chi \in \operatorname{Irr}_{S}(G)$. We define $\delta_{\chi}: Z(F[C]) \rightarrow F$ by setting $\delta_{\chi}(\widehat{K \cap C})$ $=(\chi(x)|K| / \chi(1))^{*}$ for $K S$-invariant conjugacy class of $G$ and $x \in K \cap C$. Then $\delta_{\chi}$ is an algebra homomorphism.

Proof. Since $\delta_{\chi}(\widehat{K \cap C})=\lambda_{\chi}(\widehat{K})$, it suffices to show that

$$
\delta_{\chi}\left(\widehat{K_{i} \cap} C \widehat{K_{j} \cap} C\right)=\lambda_{\chi}\left(\widehat{K}_{i} \widehat{K}_{j}\right)
$$

for $K_{i}, K_{j}, S$-invariant conjugacy classes of $G$.

Write $K_{1}, \ldots, K_{h}$ for the $S$-invariant conjugacy classes, and $K_{h+1,1}, \ldots$, $K_{h+1, a_{1}}, \ldots, K_{h+t, 1}, \ldots, K_{h+t, a_{t}}$ for the rest, where $K_{h+j, 1}, \ldots, K_{h+j, a}$ is an $S$ - orbit. Note that $a_{j}=p^{b_{j}}>1$.

Received by the editors January 15, 1988 and, in revised form, August 1, 1988.

1980 Mathematics Subject Classification (1985 Revision). Primary $20 \mathrm{C} 15$.

Key words and phrases. The Glauberman correspondence. 
It is clear that we can write

$$
\widehat{K}_{i} \widehat{K}_{j}=\sum_{k=1, \ldots, h} a_{i j k} K_{k}+\sum_{l=1, \ldots, t} b_{i j l}\left(\sum_{m=1, \ldots, a_{l}} \widehat{K}_{h+l, m}\right) .
$$

Fix $x_{k} \in K_{k} \cap C$. Since $a_{i j k}=\left|\left\{(x, y) \in K_{i} \times K_{j}: x y=x_{k}\right\}\right|^{*}$ and $\mid\{(x, y) \in$ $\left.K_{i} \times K_{j}: x y=x_{k}\right\}|\equiv|\left\{(x, y) \in K_{i} \cap C \times K_{j} \cap C: x y=x_{k}\right\} \mid \bmod p$, we have that $\widehat{K_{i} \cap} C \widehat{K_{j} \cap} C=\sum_{k=1, \ldots, h} a_{i j k} \widehat{K_{k} \cap} C$.

Now, since $\lambda_{\chi}$ is constant over each $S$-orbit, we have

$$
\begin{aligned}
\lambda_{\chi}\left(\widehat{K}_{i} \widehat{K}_{j}\right) & =\sum_{k=1, \ldots, h} a_{i j k} \lambda_{\chi}\left(\widehat{K}_{k}\right)+\sum_{l=1, \ldots, t} b_{i j l} p^{b_{l}} \lambda_{\chi}\left(\widehat{K}_{h+l, 1}\right) \\
& =\sum_{k=1, \ldots, h} a_{i j k} \lambda_{\chi}\left(\widehat{K}_{k}\right) \\
& =\sum_{k=1, \ldots, h} a_{i j k} \delta_{\chi}\left(K_{k} \widehat{\cap} C\right) \\
& =\delta_{\chi}\left(\sum_{k=1, \ldots, h} a_{i j k} \widehat{K_{k} \cap} C\right)=\delta_{\chi}\left(\widehat{K_{i} \cap} C \widehat{K_{j} \cap} C\right)
\end{aligned}
$$

The following result is well known. We give the proof to make it clear that no results on $p$-blocks are needed for this paper.

2.2. Lemma. Suppose $p$ does not divide $|G|$. The maps $\lambda_{\chi}$ for $\chi \in \operatorname{Irr}(G)$ are distinct and are all the algebra homomorphisms from $Z(F[G])$ to $F$.

Proof. $F[G]$ is a direct sum of full matrix rings over $F$, so $Z(F[G]) \cong F^{k}$ where $k=\operatorname{cl}(G) \mid$. There are thus $k$ algebra homomorphisms $Z(F[G]) \rightarrow F$ and it is enough to show that the $\lambda_{\chi}$ are distinct.

Let $e_{\chi}=\chi(1) \sum_{g \in G} \chi\left(g^{-1}\right)^{*} g \in Z(F[G])$. Then $\lambda_{\chi}\left(e_{\chi}\right)=|G|^{*} \neq 0$ and $\lambda_{\psi}\left(e_{\chi}\right)=0$ for $\chi \neq \psi$. The result now follows.

\section{The Glauberman correspondence}

Notation. Given $\chi \in \operatorname{Irr}_{S}(G)$, since $\delta_{\chi}$ is an algebra homomorphism $Z(F[C]) \rightarrow$ $F$, it follows that there exists a unique $\tilde{\chi} \in \operatorname{Irr}(C)$ such that $\delta_{\chi}=\lambda_{\tilde{\chi}}$. Thus for $x \in C$, we have

$$
(\chi(x)|K| / \chi(1))^{*}=(\tilde{\chi}(x)|K \cap C| / \tilde{\chi}(1))^{*}, \quad \text { where } K=C l_{G}(x) .
$$

Since $|K| \equiv|K \cap C| \not \equiv 0 \bmod p$, this gives $\tilde{\chi}(1) \chi(x) \equiv \chi(1) \tilde{\chi}(x) \bmod M$, for all $x \in C$.

3.1. Theorem. The map $\operatorname{Irr}_{S}(G) \rightarrow \operatorname{Irr}(C)$ defined by $\chi \rightarrow \tilde{\chi}$ is a bijection. Also, $\left[\chi_{C}, \tilde{\chi}\right] \equiv \pm 1 \bmod p$, and $\left[\chi_{C}, \theta\right] \equiv 0 \bmod p$ for $\tilde{\chi} \neq \theta \in \operatorname{Irr}(C)$.

Proof. Let $\chi \in \operatorname{Irr}_{S}(G)$ and $\theta \in \operatorname{Irr}(C)$. Then

$$
\begin{aligned}
|C| \tilde{\chi}(1)\left[\chi_{C}, \theta\right]=\tilde{\chi}(1) \sum_{x \in C} \chi(x) \theta\left(x^{-1}\right) \equiv \chi(1) \sum_{x \in C} \tilde{\chi}(x) \theta\left(x^{-1}\right) & \\
=\chi(1)[\tilde{\chi}, \theta]|C| & \bmod M .
\end{aligned}
$$


Since $p$ does not divide $|C|$, this gives $\tilde{\chi}(1)\left[\chi_{C}, \theta\right] \equiv \chi(1)[\tilde{\chi}, \theta] \bmod p$. Since $p$ does not divide $\tilde{\chi}(1)$, taking $\theta \neq \tilde{\chi}$, this gives $\left[\chi_{C}, \theta\right] \equiv 0 \bmod p$, and taking $\theta=\tilde{\chi}$ gives

$$
\tilde{\chi}(1)\left[\chi_{C}, \tilde{\chi}\right] \equiv \chi(1) \not \equiv 0 \bmod p .
$$

Thus $\tilde{\chi}$ is the unique irreducible consituent of $\chi_{C}$ with multiplicity not divisible by $p$.

Now let $\chi, \varphi \in \operatorname{Irr}_{S}(G)$. Then

$$
|G|[\chi, \varphi]=\sum_{x \in G} \chi(x) \varphi\left(x^{-1}\right) \equiv \sum_{x \in C} \chi(x) \varphi\left(x^{-1}\right)=|C|\left[\chi_{C}, \varphi_{C}\right] \quad \bmod M
$$

(using that $\chi, \varphi$ are $S$-invariant). Also, $|G| \equiv|C| \not \equiv 0 \bmod p$, and so $[\chi, \varphi] \equiv$ $\left[\chi_{C}, \varphi_{C}\right] \bmod p$.

Since $\chi_{C}=\left[\chi_{C}, \tilde{\chi}\right] \tilde{\chi}+p \Delta$ and $\varphi_{C}=\left[\varphi_{C}, \tilde{\varphi}\right] \tilde{\varphi}+p E$, we have that $[\chi, \varphi]=$ $\left[\chi_{C}, \tilde{\chi}\right]\left[\varphi_{C}, \tilde{\varphi}\right][\tilde{\chi}, \tilde{\varphi}] \bmod p .(2)$

This shows that our map is injective.

If $\alpha \in \operatorname{Irr}(C)$ is not in the image of our map, then $\left[\chi_{C}, \alpha\right]=\left[\chi, \alpha^{G}\right] \equiv$ $0 \bmod p$ for all $\chi \in \operatorname{Irr}_{S}(G)$. Since $\alpha^{G}$ is $S$-invariant, $\left[\alpha^{G}, \varphi^{s}\right]=\left[\alpha^{G}, \varphi\right]$ for all $\varphi \in \operatorname{Irr}(G)$, for all $s \in S$. This implies that $p$ divides $\alpha^{G}(1)$, a contradiction.

Finally, taking $\chi=\varphi$ in (2), we get $1 \equiv\left[\chi_{C}, \tilde{\chi}\right]^{2} \bmod p$ and so $\left[\chi_{C}, \tilde{\chi}\right] \equiv$ $\pm 1 \bmod p$ as claimed.

\section{ACKNOWLEDGMENT}

The author wishes to express his gratitude to Professor I. M. Isaacs for his invaluable advice.

\section{REFERENCES}

1. G. Glauberman, Correspondences of characters for relatively prime operator groups, Canad. J. Math. 20 (1968), 1465-1488.

2. I. M. Isaacs, Character theory of finite groups, Academic Press, New York, 1976.

Departamento de Algebra, Facultad de Ciencias Matemáticas, Universitat de Valencia, c/o Doctor Moliner, 50 Burjasot, Valencia, Spain 